\title{
CORRESPONDENCE
}

\section{Targeting monoamine oxidase to dampen NLRP3 inflammasome activation in inflammation}

\author{
Ricardo Sánchez-Rodríguez ${ }^{1,2}$, Fabio Munari ${ }^{1,2}$, Roberta Angioni ${ }^{2}$, Francisca Venegas ${ }^{1,2}$, Andrielly Agnellini ${ }^{1,3}$, \\ María Paulette Castro-Gil (iD ${ }^{1,2,4}$, Alessandra Castegna ${ }^{5}$, Roberto Luisetto ${ }^{6}$, Antonella Viola ${ }^{1,2}$ and Marcella Canton ${ }^{1,2}$
}

Cellular \& Molecular Immunology_\#\#\#\#\#\#\#\#\#\#\#\#\#\#\#\#\#_; https://doi.org/10.1038/s41423-020-0441-8

Inflammasomes represent protective weapons against pathogens and cellular damage, although their uncontrolled activation drives the progression of inflammatory, metabolic, and neurodegenerative disorders. Several signals activate the NLRP3 inflammasome, and a few studies have reported that mitochondrial reactive oxygen species (ROS) are involved in this process. However, the source of mitochondrial ROS and their specific role in NLRP3 triggering are unclear. Here, we show that $\mathrm{H}_{2} \mathrm{O}_{2}$ produced by the mitochondrial enzyme monoamine oxidase $B$ (MAO-B) plays a nonredundant role in sustaining NLRP3 inflammasome activation. Mechanistically, MAO-B-dependent ROS formation caused mitochondrial dysfunction and NF-KB induction, resulting in NLRP3 and pro-IL-1 $\beta$ overexpression. Both in vitro and in vivo, MAO-B inhibition by rasagiline prevented IL-1 $\beta$ secretion, and MAO-Bdeficient mice showed an impaired response to LPS-mediated endotoxemia. Our findings identify MAO-B as a specific producer of mitochondrial ROS fueling the NLRP3 inflammasome, thereby providing a basis for repurposing $\mathrm{MAOB}$ inhibitors to treat inflammasome-mediated pathologies.

Inflammation is a complex process orchestrated by the immune system in response to infection or tissue injury. Pathogenassociated molecular patterns (PAMPs) or danger-associated molecular patterns (DAMPs) are sensed by innate immune receptors, which trigger signaling pathways leading to the release of pro-inflammatory cytokines. The NLRP3 inflammasome, a key sensor of these signals, is a multiprotein platform generating active caspase- 1 , which converts IL-1 $\beta$ and IL-18 precursors into their mature active forms. ${ }^{1}$ NLRP3 inflammasome assembly results from a two-step process. The priming signal is induced by the tolllike receptor (TLR)/nuclear factor (NF)-KB pathway and upregulates the expression of proteins involved in the process, including NLRP3.' The second signal results in the assembly of the multiprotein, active complex and seems to involve alterations in ion homeostasis, lysosomal rupture and mitochondrial ROS generation. ${ }^{1}$

ROS are known to tune NLRP3 priming by inducing NF-KB activation. However, the molecular source of NLRP3-triggered ROS is still unclear. Some studies highlighted that mitochondria are crucial in activating the NLRP3 inflammasome ${ }^{2}$ and that mitophagy/autophagy blockade leads to the accumulation of damaged mitochondria and inflammasome activation.,4 Mitochondria represent a relevant source of ROS, as they produce these species through several mechanisms, including electron leakage to oxygen at complex I or III of the respiratory chain. Among the enzymes capable of ROS formation, two isoforms of NADPH oxidase (NOX2 and NOX4) were identified in phagocytes, but their role in NLRP3 inflammasome activation is controversial. ${ }^{5,6}$ A relevant mitochondrial-specific source of ROS is monoamine oxidase (MAO), although its impact on inflammation has been quite overlooked. MAO catalyzes the oxidative deamination of neurotransmitters and dietary amines, generating aldehydes, ammonia and $\mathrm{H}_{2} \mathrm{O}_{2}{ }^{7}$. The two isoforms, MAO-A and MAO-B, differ in substrate specificity and inhibitor sensitivity. However, they show similar affinity for dopamine and tyramine. The physiologic role of MAO enzymes is well established in the central nervous system: they terminate neurotransmitter signaling, thereby generating $\mathrm{H}_{2} \mathrm{O}_{2}$ that is removed by endogenous scavengers. In pathological conditions, the increased activity of MAO enzymes overcomes cellular antioxidant defenses, altering redox homeostasis and eliciting deleterious effects, as in muscular dystrophy. ${ }^{8}$ Considering the importance of identifying and targeting NLRP3 activators, we investigated whether MAO-B regulates inflammasome triggering and can thus represent a novel therapeutic target to control inflammation.

With this aim, murine bone marrow-derived macrophages (BMDMs) were triggered by LPS/ATP in the presence or absence of rasagiline, a clinical-grade MAO-B inhibitor. The effect of MAO-B inhibition on ROS production and mitochondrial depolarization, hallmarks of NLRP3 inflammasome activation, ${ }^{2,3}$ was evaluated. Rasagiline suppressed ROS production (Fig. 1a) and prevented mitochondrial depolarization (Fig. 1b) in response to stimuli inducing inflammasome activation. Moreover, rasagiline significantly reduced IL-1 $\beta$ secretion (Fig. $1 c$ ) and ASC speck formation (Fig. 1d) in stimulated BMDMs, but had no effect on the production of the inflammasome-independent cytokine tumor necrosis alpha (TNF-a) (Fig. 1e). Similar results were obtained using human monocyte-derived macrophages (Fig. S1).

LPS/ATP stimulation elicited an increase in MAO-B protein levels (Fig. S2a) and activity (Fig. S2b). More specifically, LPS/ATP caused a rise in the extracellular levels of the MAO substrate dopamine, which further increased when its consumption was blocked by MAO inhibition (Fig. S2b), suggesting possible control of inflammasome activation, as previously described. ${ }^{9}$

ROS may regulate NLRP3 priming through NF-KB, which induces the expression of inflammasome components. Rasagiline dampened LPS/ATP-induced NF-KB activation (Fig. 1f) and reduced

\footnotetext{
${ }^{1}$ Department of Biomedical Sciences, University of Padova, 35131 Padova, Italy; ${ }^{2}$ Fondazione Istituto di Ricerca Pediatrica Città della Speranza - IRP, 35129 Padova, Italy; ${ }^{3}$ Venetian Institute of Molecular Medicine, Padova, Italy; ${ }^{4}$ Laboratorio de Enfermedades Hepáticas, Instituto Nacional de Medicina Genómica, CDMX, Mexico; ${ }^{5}$ Department of Biosciences, Biotechnologies and Biopharmaceutics, University of Bari, Bari, Italy and ${ }^{6}$ Department of Surgery, Oncology and Gastroenterology, University of Padova, Padova, Italy Correspondence: Antonella Viola (antonella.viola@unipd.it) or Marcella Canton (marcella.canton@unipd.it)
}

Received: 7 April 2020 Accepted: 8 April 2020

Published online: 28 April 2020 


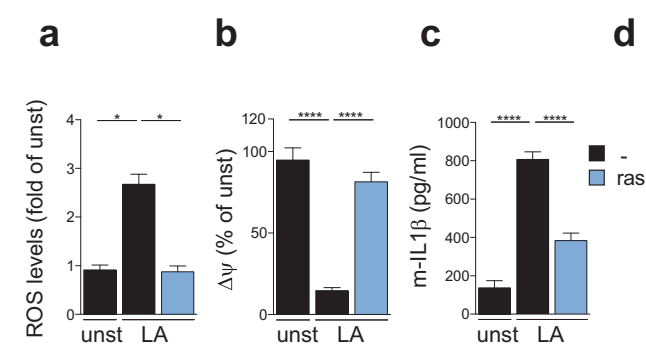

e

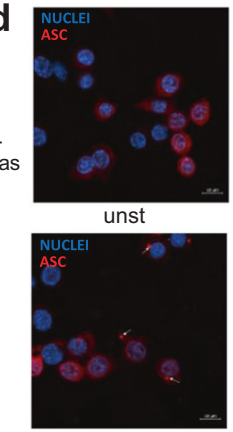

ras $10+\mathrm{LA}$

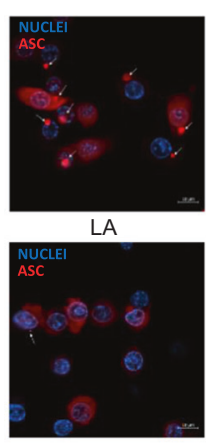

ras $20+$ LA

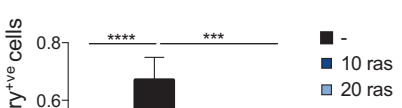

g

h

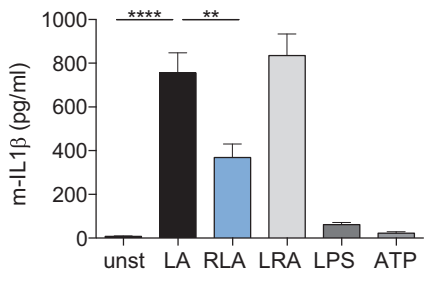

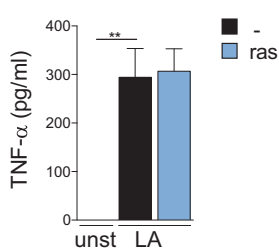
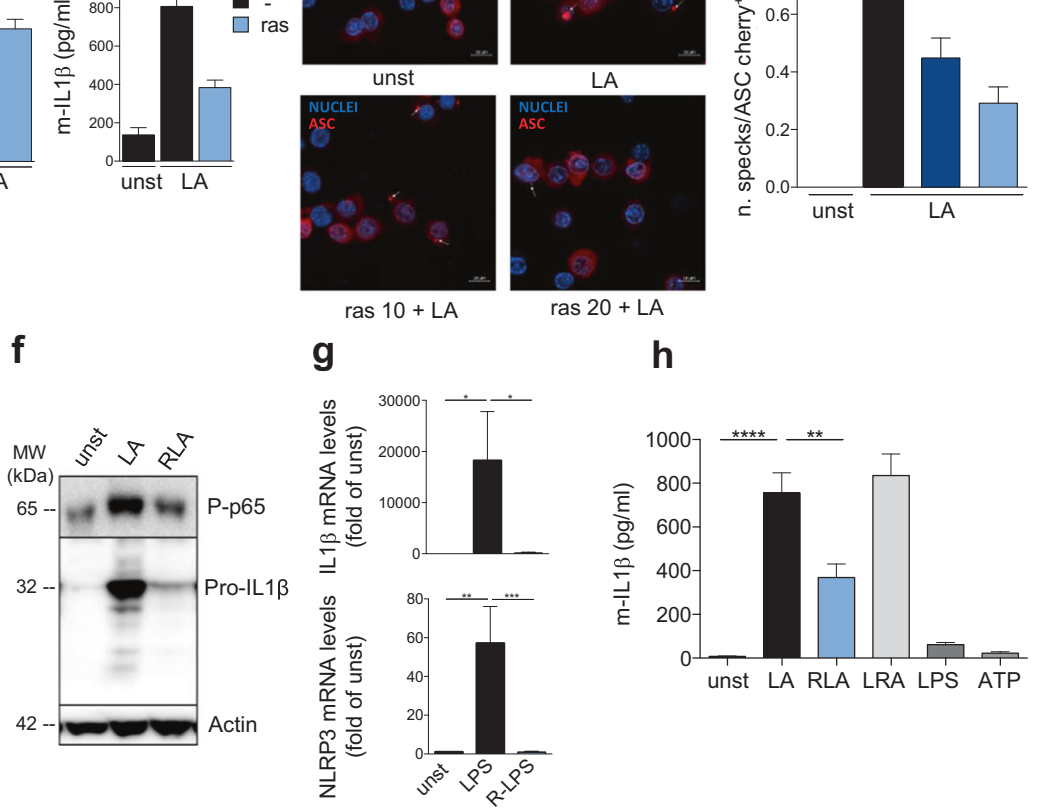

10 ras

-

i
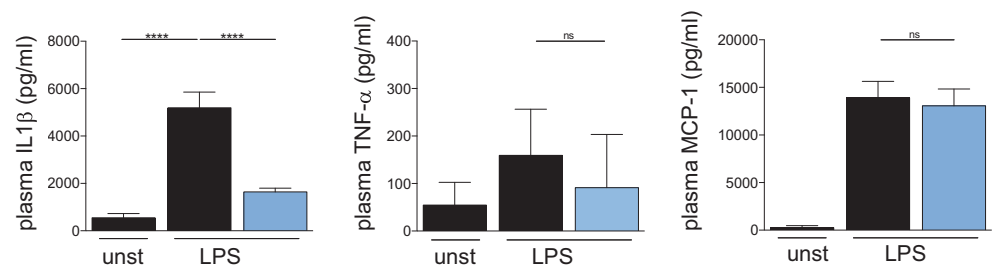

j
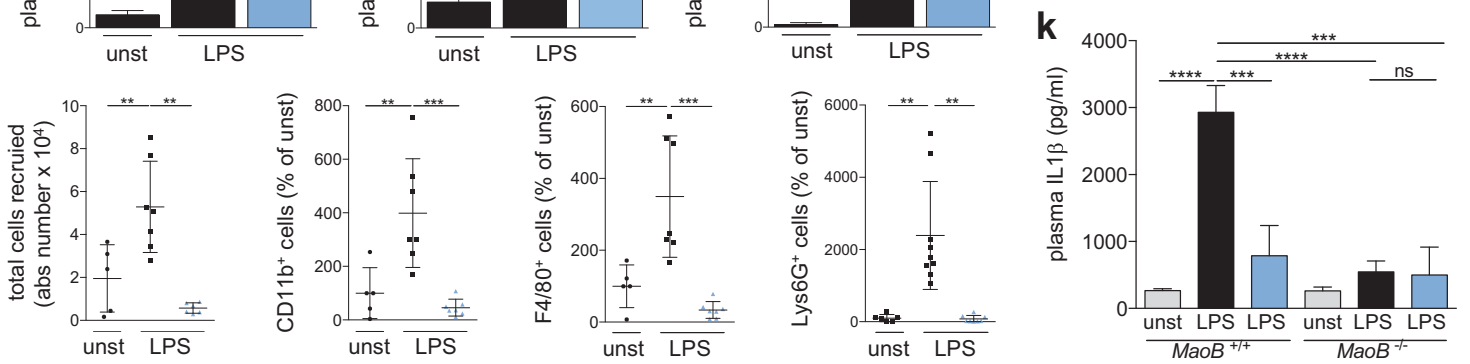

Fig. 1 a ROS levels detected in BMDMs stained with MitoSOX (2.5 $\mu \mathrm{M})$ after NLRP3 inflammasome activation by LPS (100 ng/ml, $2 \mathrm{~h}$ ) and ATP $(5 \mathrm{mM}$, last $15 \mathrm{~min})(\mathrm{LA})$ with or without rasagiline pretreatment (ras, $20 \mu \mathrm{M})$. Values are reported as the fold-changes from of unstimulated cells (unst) ( $N=3$ independent experiments). b Mitochondrial membrane potential $(\Delta \Psi)$ was measured with TMRM (50 nM) by fluorescence microscopy in BMDMs after NLRP3 inflammasome activation by LPS $(100 \mathrm{ng} / \mathrm{ml}, 4 \mathrm{~h}$ ) and ATP $(5 \mathrm{mM}$, last $15 \mathrm{~min})$ (LA) with or without rasagiline pretreatment $(\mathrm{ras}, 20 \mu \mathrm{M})$. Images were acquired before and after FCCP $(4 \mu \mathrm{M})$ addition. The graph reports the difference in fluorescence intensities before and after FCCP. Values are reported as percentages relative to unstimulated cells $(N=3$ independent experiments). c ELISA of IL-1 $\beta$ in supernatants from BMDMs treated as in (b) ( $N=6$ independent experiments). d Representative images (left) and quantification (right) of ASC specks in ASC-mCherry macrophages treated as in (b) in the absence or presence of rasagiline (10-20 $\mu \mathrm{M})$ $(N=3$ independent experiments). The number of ASK specks was normalized to total ASC-mCherry-positive macrophages. e ELISA of TNF- $\alpha$ in supernatants from BMDMs treated as in (b) $(N=5$ independent experiments). $f$ Western blots for IL- $1 \beta$ and NF- $\kappa B$ (the active form phosphop65, P-p65) in total protein lysates from BMDMs treated as in (b) (representative of three independent experiments). $\beta$-Actin was used as a loading control. g BMDMs either treated or untreated with rasagiline $(R, 20 \mu M)$ were stimulated for $2 \mathrm{~h}$ with LPS $(100 \mathrm{ng} / \mathrm{ml})$. Rasagiline decreased the mRNA expression of $I L-1 \beta$ and NIrp3 as measured by RT-PCR in real time using GAPDH as a housekeeping control ( $N=4$ independent experiments). $\mathbf{h}$ BMDMs were exposed to the following protocols: LPS $(100 \mathrm{ng} / \mathrm{ml}, 4 \mathrm{~h})$ and ATP (5 mM, last $15 \mathrm{~min})$ treatment (LA); 15 -min pretreatment with rasagiline $(20 \mu \mathrm{M})$ and subsequent treatment with LA (RLA); LPS treatment (100 ng/ml) followed by rasagiline treatment $(20 \mu \mathrm{M}) 15 \mathrm{~min}$ before ATP treatment $(5 \mathrm{mM})$ (LRA); LPS treatment alone; or ATP treatment alone; unstimulated cells were used as controls (LPS, ATP and unst, respectively). IL-1 $\beta$ levels released in supernatants were measured by ELISA ( $N=5$ independent experiments). i ELISA of plasma IL-1 $\beta$, TNF- $\alpha$ and MCP-1 from C57BL6 mice orally treated with vehicle or rasagiline $(0.5 \mathrm{mg} / \mathrm{kg})$. After $15 \mathrm{~h}$, mice were intraperitoneally injected with LPS $(10 \mathrm{mg} / \mathrm{kg})$ or PBS (LPS, $n=7$; LPS + ras, $n=7$; PBS, $n=5, N=3$ independent experiments). Mice were sacrificed after $8 \mathrm{~h}$. $\mathbf{j}$ Cells derived from the peritoneal cavity of the mice described in (i) were stained and analyzed by flow cytometry. $\mathbf{k}$ MAOB-deficient mice and their wild-type littermates were orally treated with vehicle or rasagiline $(0.5 \mathrm{mg} / \mathrm{kg})$. After $15 \mathrm{~h}$, they were intraperitoneally injected with LPS $(10 \mathrm{mg} / \mathrm{kg})$ or PBS (LPS, $n=6$; LPS + ras, $n=4$; PBS, $n=5$ for MAO-B KO mice, LPS, $n=4$; LPS + ras, $n=3$; PBS, $n=3$ for WT mice, $N=2$ independent experiments). Mice were sacrificed after $8 \mathrm{~h}$, and IL-1 $\beta$ plasma levels were measured by ELISA. Data are presented as the mean \pm SEM. Statistical analysis was performed with one-way ANOVA with Tukey's post hoc test (a-c and $n)$ and the Kruskal-Wallis test with Dunn's post hoc test $(\mathbf{d}-\mathbf{m}){ }^{*} P<0.05$, ${ }^{* *} P<0.01,{ }^{* *} P<0.005,{ }^{* * *} P<0.001$ 
pro-IL-1 $\beta$ and NLRP3 expression (Fig. 1g). To gain more insight into the mechanism of action of MAO-B in NLRP3 inflammasome triggering, we analyzed the effect of rasagiline on the NLRP3 priming and activation steps. We found that rasagiline affected IL$1 \beta$ secretion only when added before LPS stimulation, indicating that MAO-B is required for the priming step of NLRP3 activation (Fig. 1h). Altogether, these data indicate that MAO-B activity is required for the generation of ROS that fuel the inflammasome through an NF-KB-mediated mechanism.

To test the impact of MAO-B inhibition in vivo, we challenged mice with intraperitoneal LPS injection and analyzed the levels of circulating IL-1 $\beta$, TNF- $a$, and monocyte chemoattractant protein-1 (MCP-1), along with the number of cells infiltrating the peritoneal cavity. We found that rasagiline pretreatment reduced IL-1 $\beta$ production in response to LPS stimulation (Fig. 1i) and dampened the recruitment of inflammatory cells into the peritoneal cavity (Fig. 1j, Fig. S3). In contrast, rasagiline did not modify the secretion of TNF- $\alpha$ and MCP-1 (Fig. 1i). To validate the target of rasagiline by a genetic approach, we used MAO-B-deficient mice (Fig. S4) challenged with LPS injection. MAO-B KO mice had a milder response to LPS challenge than MAO-B wild-type mice, as detected by IL-1 $\beta$ plasma levels, and rasagiline treatment in these mice did not provide further IL-1 $\beta$ decrease (Fig. $1 \mathrm{k}$ ).

Therapies targeting the NLRP3 inflammasome are urgently needed since its excessive activation has been related to several disorders, and indeed, several inhibitors are under investigation. ${ }^{10}$ In this context, our results that identify MAO-B as a crucial, nonredundant source of ROS in NLRP3 inflammasome activation suggest that iMAO-B may represent very attractive drugs.

Mechanistically, we found that MAO-B expression is induced by NLRP3 stimuli, which also cause an increase in the levels of the MAO substrate dopamine. MAO-B activity is therefore potentiated during NLRP3 stimulation, resulting in increased $\mathrm{H}_{2} \mathrm{O}_{2}$ production. Our results indicate that in macrophages stimulated by NLRP3inducing stimuli, MAO-B-derived ROS are necessary to sustain IL$1 \beta$ secretion through a pathway involving NF-KB and thus affect the NLRP3 inflammasome priming step.

These findings may open the way for new therapeutic approaches to inflammatory diseases based on iMAO-B. The fact that MAO-B inhibition prevents mitochondrial dysfunction, the accumulation of damaged mitochondria, and, potentially, mitochondrial DAMP release gives it a great advantage over other compounds. ${ }^{3,4}$ Moreover, MAO-B inhibition should prevent the formation of the specific subset of mitochondrial ROS that becomes excessive under pathological conditions, in contrast to the general antioxidants that remove ROS in an unspecific manner, thus affecting also fundamental ROS-mediated functions.

Moreover, from a translational point of view, there are several advantages in MAO-B targeting because iMAO-B are well tolerated, and rasagiline is already used to treat Parkinson's disease. We believe that repurposing iMAO-B would allow a fast and safe approach to treat several inflammatory diseases characterized by IL-1 $\beta$-driven pathology.

\section{ACKNOWLEDGEMENTS}

We thank P. Pelegrin (Instituto Murciano de Investigación Biosanitaria) and E. Latz (Institute of Innate Immunity) for the immortalized ASC-mCherry macrophages. We acknowledge Paola Brun, Ignazio Castagliuolo, Bianca Cali, Sara Zumerle, and Denis Martin-Valet for helpful discussion and Luisa Bin and Alberto Zecca for their contribution in performing experiments. The study was supported by ERC Steps 322823 to A.V. and IRP-PENTA Grant 18/07-1 to M.C.

\section{AUTHOR CONTRIBUTIONS}

A.V. and M.C. designed the study, wrote the manuscript, and provided funds; R.S.R., F. M., A.A., F.V., and R.A. performed most of the experiments and the subsequent data analyses and prepared the figures; M.P.C.G. helped in performing experiments; R.L. performed in vivo experiments in the murine endotoxemia model; A.C. performed mass spectrometric analyses.

\section{ADDITIONAL INFORMATION}

The online version of this article (https://doi.org/10.1038/s41423-020-0441-8) contains supplementary material.

Competing interests: The authors declare no competing interests.

\section{REFERENCES}

1. Swanson, K. V. et al. The NLRP3 inflammasome: molecular activation and regulation to therapeutics. Nat. Rev. Immunol. 19, 477-489 (2019).

2. Zhou, R. et al. A role for mitochondria in NLRP3 inflammasome activation. Nature 469, 221-225 (2011).

3. Zhong, Z. et al. NF-KB restricts inflammasome activation via elimination of damaged mitochondria. Cell 164, 896-910 (2016).

4. Afonina, I. S. et al. Limiting inflammation - The negative regulation of NF-KB and the NLRP3 inflammasome. Nat. Immunol. 18, 861-869 (2017).

5. Van Bruggen, R. et al. Human NLRP3 inflammasome activation is Nox1-4 independent. Blood 115, 5398-5400 (2010).

6. Moon, J. S. et al. NOX4-dependent fatty acid oxidation promotes NLRP3 inflammasome activation in macrophages. Nat. Med. 22, 1002-1012 (2016).

7. Youdim, M. B. H. et al. The therapeutic potential of monoamine oxidase inhibitors. Nat. Rev. Neurosci. 7, 295-309 (2006).

8. Menazza, S. et al. Oxidative stress by monoamine oxidases is causally involved in myofiber damage in muscular dystrophy. Hum. Mol. Genet 19, 4207-4215 (2010).

9. Yan, Y. et al. Dopamine controls systemic inflammation through inhibition of NLRP3 inflammasome. Cell 160, 62-73 (2015).

10. Mangan, M. S. J. et al. Targeting the NLRP3 inflammasome in inflammatory diseases. Nat. Rev. Drug Discov. 17, 688 (2018). 